\title{
Effect of meal timing and glycaemic index on glucose control and insulin secretion in healthy volunteers
}

\author{
Linda M. Morgan ${ }^{1}$, Jiang-Wen Shi ${ }^{1}$, Shelagh M. Hampton ${ }^{1}$ and Gary Frost ${ }^{2 *}$ \\ ${ }^{1}$ Faculty of Health and Medical Sciences, University of Surrey, Guildford GU2 7XH, UK \\ ${ }^{2}$ Nutrition and Dietetic Research Group, Investigative Science, Gth Floor Commonwealth Building, Faculty of Medicine, \\ Imperial College, Hammersmith Campus, DuCane Road, London W12 ONN, UK
}

(Submitted 11 July 2011 - Final revision received 24 October 2011 - Accepted 29 October 2011 - First published online 16 December 2011)

\begin{abstract}
Shiftworkers have a higher risk of CHD and type 2 diabetes. They consume a large proportion of their daily energy and carbohydrate intake in the late evening or night-time, a factor which could be linked to their increase in disease risk. We compared the metabolic effects of varying both dietary glycaemic index (GI) and the time at which most daily energy intake was consumed. We hypothesised that glucose control would be optimal with a low-GI diet, consumed predominantly early in the day. A total of six healthy lean volunteers consumed isoenergetic meals on four occasions, comprising either high- or low-GI foods, with $60 \%$ energy consumed predominantly early (breakfast) or late (supper). Interstitial glucose was measured continuously for $20 \mathrm{~h}$. Insulin, TAG and non-esterified fatty acids were measured for $2 \mathrm{~h}$ following every meal. Highest glucose values were observed when large $5021 \mathrm{~kJ}$ (1200 kcal) high-GI suppers were consumed. Glucose levels were also significantly higher in predominantly late high- $v$. low-GI meals $(P<0 \cdot 01)$. Using an estimate of postprandial insulin sensitivity throughout the day, we demonstrate that this follows the same trend, with insulin sensitivity being significantly worse in high energy consumed in the evening meal pattern. Both meal timing and GI affected glucose tolerance and insulin secretion. Avoidance of large, highGI meals in the evening may be particularly beneficial in improving postprandial glucose profiles and may play a role in reducing the risk of type 2 diabetes; however, longer-term studies are needed to confirm this.
\end{abstract}

Key words: Meal timing: Glycaemic index: Glucose: Insulin

The level of postprandial blood glucose is an independent risk factor for the future development of type 2 diabetes ${ }^{(1)}$. Shiftworkers have a higher risk of $\mathrm{CHD}^{(2)}$ and type 2 diabetes $^{(3)}$. They consume a large proportion of their daily energy and carbohydrate intake in the late evening or night-time, a factor which could be linked to their increase in disease risk. There is a diurnal variation in insulin resistance, with the greatest insulin resistance found at night ${ }^{(4)}$. We have demonstrated previously a relatively impaired postprandial glucose and lipid tolerance following meals eaten at night, compared with identical meals consumed during the day ${ }^{(5)}$. In Western societies, most energy intake now occurs in the evening.

We have also demonstrated that a low-glycaemic index (LGI) diet reduces postprandial blood glucose, at the second meal and over the $24 \mathrm{~h}$ period, suggesting that LGI may help in the prevention of the metabolic syndrome ${ }^{(6)}$.
We hypothesise that a large evening energy and carbohydrate load will cause an increase in postprandial glucose compared to the same in the morning and that the postulated high glycaemic excursions in the evening could be ameliorated by decreasing the glycaemic index (GI) of the meal.

\section{Experimental methods}

\section{Participants}

A total of six healthy volunteers (four females, two males) were recruited (mean age 30 (SEM 4.3) years, BMI 21.6 (SEM 1.3$) \mathrm{kg} / \mathrm{m}^{2}$ ). The present study was conducted according to the guidelines laid down in the Declaration of Helsinki; and the experimental protocols and procedures involving human subjects were approved by the University of Surrey Ethics Committee (IC/2006/23/SBMS). Written informed consent was obtained from all subjects. The study was carried out in

Abbreviations: GI, glycaemic index; HGI-am, high glycaemic index, with majority of energy load consumed in the morning; HGI-pm, high glycaemic index, with majority of energy load consumed in the evening; LGI-am, low glycaemic index, with majority of energy load consumed in the morning; LGI-pm, low glycaemic index, with majority of energy load consumed in the evening.

* Corresponding author: Professor G. Frost, fax + 44208383 8320, email g.frost@imperial.ac.uk 
the Clinical Investigation Unit (CIU) at Surrey University. We used a four-way randomised crossover study methodology as outlined next.

\section{Diets}

There were four diets:

(1) Low GI (average GI = 34), with the majority of energy load consumed in the morning (LGI-am)

(2) Low GI, with the majority of energy load consumed in the evening (LGI-pm)

(3) High GI (average GI = 84), with the majority of energy load consumed in the morning (HGI-am)

(4) High GI, with the majority of energy load consumed in the evening (HGI-pm)

The volunteers were randomised to each diet using random number allocation carried out independently of the study personnel. Each diet had a total energy content of approximately $8368 \mathrm{~kJ}$ (2000 kcal) for the whole day and had identical macronutrient content. However, as would be expected, the fibre intake was higher and the glycaemic load lower in the lowGI diets. For the morning load diet, energy was split $60 \%$ $(5021 \mathrm{~kJ}(1200 \mathrm{kcal}))$ at breakfast, $20 \%(1674 \mathrm{~kJ}(400 \mathrm{kcal}))$ at lunch and $20 \%(1674 \mathrm{~kJ}$ (400 kcal)) for the dinner (Tables 1 and 2). This was reversed on the evening load diet. This approach decreased variation due to change in type of food. Breakfast was given at 09.30 hours, lunch at 13.30 hours and the evening meal at 20.30 hours. All volunteers completed the four interventions, with a minimum of $7 \mathrm{~d}$ between interventions. The GI values of the mixed meal in the experimental diets were calculated according to the calibration formula derived by Wolever ${ }^{(7)}$. The glycaemic load was calculated by multiplying the GI by the dietary carbohydrate of the individual food divided by $100^{(8)}$. All foods were bought from a single supplier, and all diets were prepared on the study day.

\section{Blood sampling}

Participants were asked to fast after supper the night before each study day until the following morning and avoid alcohol or heavy exercise. They attended the clinical investigation unit at 08.00 hours. The study was conducted with the volunteers seated throughout the day, with movement kept to a minimum. For each study day, finger-prick capillary blood samples were taken before and at 30,60 and 120 min following every meal. Blood was taken using preset lancets and collected in fluoride oxalate tubes by the participants themselves. A $300 \mu \mathrm{l}$ sample was used to analyse for blood glucose, insulin, NEFA and TAG. Once the blood samples were collected, they were centrifuged in a refrigerated centrifuge for $10 \mathrm{~min}$ at $2000 \mathrm{~g}$ to harvest the plasma. The glucose measurements were carried out directly on plasma on the same day using the YSI 2300 STAT plus analyser (YSI Life Sciences) and then the plasma samples were stored at $-80^{\circ} \mathrm{C}$ until required.

\section{Continuous glucose monitoring}

On the day before the study day, between 15.00 and 16.00 hours, participants attended the CIU, where they were fitted with the MiniMed continuous glucose monitoring system

Table 1. Nutritional breakdown of the high-glycaemic index (GI) diet used in the study*

\begin{tabular}{|c|c|c|c|c|c|c|c|c|}
\hline & \multicolumn{8}{|c|}{ High GI (am) } \\
\hline & Weight (g) & Energy (kJ) & Fat $(\mathrm{g})$ & Protein $(\mathrm{g})$ & Carbohydrate (g) & Fibre $(\mathrm{g})$ & Gl & $\mathrm{GL}$ \\
\hline \multicolumn{9}{|l|}{ Breakfast } \\
\hline Fruit loaf & 144 & 1674 & 6 & 10 & 77 & 5 & 90 & 64 \\
\hline Margarine & 25 & 799 & 19 & 0 & 0 & 0 & & \\
\hline Skimmed milk & 150 & 234 & 1 & 5 & 7 & 0 & 48 & 4 \\
\hline Cheese, cheddar & 30 & 523 & 10 & 8 & 0 & 0 & 0 & 0 \\
\hline Strawberry low fat yoghurt & 125 & 418 & 1 & 3 & 20 & 1 & 85 & 16 \\
\hline Mars bar & 54 & 1029 & 10 & 2 & 38 & 0 & 68 & 26 \\
\hline Water & 200 & 0 & 0 & 0 & 0 & 0 & 0 & 0 \\
\hline Glucose & 15 & 247 & 0 & 0 & 16 & 0 & 100 & 16 \\
\hline Total & & 4925 & 46 & 28 & 157 & 6 & 83 & 126 \\
\hline \multicolumn{9}{|l|}{ Lunch } \\
\hline Strawberry low fat yoghurt & 125 & 418 & 1 & 3 & 20 & 1 & 85 & 16 \\
\hline Cottage pie & 300 & 1004 & 8 & 15 & 26 & 4 & 65 & 15 \\
\hline Water & 200 & 0 & 0 & 0 & 0 & 0 & & 0 \\
\hline Glucose & 15 & 247 & 0 & 0 & 16 & 0 & 100 & 16 \\
\hline Total & & 1669 & 9 & 18 & 62 & 4 & 80 & 47 \\
\hline \multicolumn{9}{|l|}{ Dinner } \\
\hline Sultana Bran & 90 & 1226 & 2 & 7 & 61 & 12 & 90 & 45 \\
\hline Skimmed milk & 150 & 234 & 1 & 5 & 7 & 0 & 48 & 4 \\
\hline Water & 20 & 0 & 0 & 0 & 0 & 0 & 0 & 0 \\
\hline Glucose & 15 & 247 & 0 & 0 & 16 & 0 & 100 & 16 \\
\hline Cheese, cheddar & 5 & 80 & 2 & 1 & 0 & 0 & 0 & 0 \\
\hline Total & & 1782 & 4 & 14 & 84 & 12 & 88 & 64 \\
\hline Total for the day & & 8376 & 59 & 59 & 304 & 22 & 84 & 236 \\
\hline
\end{tabular}

GL, glycaemic load.

* The meal pattern and the energy load given in the morning. The meal pattern when the energy load was given in the evening was a reflection of the morning meal pattern. There was a direct swap of the breakfast to dinner and the dinner to breakfast. 
Table 2. Nutritional breakdown of the low-glycaemic index (GI) diet used in the study*

\begin{tabular}{|c|c|c|c|c|c|c|c|c|}
\hline & \multicolumn{8}{|c|}{ Low GI (am) } \\
\hline & Weight (g) & Energy (kJ) & Fat $(\mathrm{g})$ & Protein $(\mathrm{g})$ & Carbohydrate (g) & Fibre $(g)$ & Gl & GL \\
\hline \multicolumn{9}{|l|}{ Breakfast } \\
\hline Pumpernickel bread & 180 & 1414 & 2 & 9 & 70 & 6 & 41 & 26 \\
\hline Black cherry yoghurt & 150 & 837 & 9 & 5 & 24 & 1 & 17 & 4 \\
\hline Semi-skimmed milk & 150 & 314 & 3 & 5 & 7 & 0 & 25 & 2 \\
\hline Apple & 133 & 268 & 0 & 0 & 15 & 3 & 38 & 5 \\
\hline Margarine & 12 & 377 & 10 & 0 & 0 & 0 & 0 & 0 \\
\hline Fruit and nut & 90 & 1715 & 24 & 6 & 42 & 5 & 15 & 6 \\
\hline Total & & 4929 & 47 & 26 & 159 & 15 & 29 & 42 \\
\hline \multicolumn{9}{|l|}{ Lunch } \\
\hline Lasagne & 280 & 1159 & 7 & 14 & 37 & 3 & 28 & 10 \\
\hline Probiotic orange drink & 200 & 544 & 2 & 4 & 23 & 1 & 30 & 7 \\
\hline Total & & 1703 & 9 & 18 & 60 & 4 & 29 & 16 \\
\hline \multicolumn{9}{|l|}{ Dinner } \\
\hline All-bran & 80 & 1092 & 1 & 8 & 56 & 23 & 42 & 14 \\
\hline Semi-skimmed milk & $150 \mathrm{ml}$ & 314 & 3 & 5 & 7 & 0 & 25 & 2 \\
\hline Orange juice & $200 \mathrm{ml}$ & 352 & 0 & 1 & 20 & 0 & 50 & 10 \\
\hline Total & & 1757 & 4 & 14 & 83 & 23 & 43 & 26 \\
\hline Total for the whole day & & 8389 & 60 & 59 & 302 & 42 & 34 & 87 \\
\hline
\end{tabular}

GL, glycaemic load.

*The meal pattern and the energy load given in the morning. The meal pattern when the energy load was given in the evening was a reflection of the morning meal pattern. There was a direct swap of the breakfast to dinner and the dinner to breakfast.

device. The MiniMed continuous glucose monitoring system senses interstitial glucose by electrochemical detection in subcutaneous interstitial fluid. It can provide an average glucose measurement every $5 \mathrm{~min}$ for up to $72 \mathrm{~h}$. A total of 288 glucose readings every $24 \mathrm{~h}$ can be obtained from the sensor system. The MiniMed continuous glucose monitoring system recorded each participant's interstitial glucose profile information until it was removed at about 11.00 hours following the study day. The same monitor was used for each subject to minimise the variations due to inter-sensor variability. This methodology had been reported by us before ${ }^{(6)}$.

\section{Postprandial insulin resistance}

An index of postprandial insulin resistance (HOMA-PP) was obtained for the three meals in each diet by using the following equation ${ }^{(6,9)}$ :

$$
\begin{aligned}
& \text { HOMA-PP } \\
& \begin{aligned}
= & \text { incremental area under the curve (IAUC) glucose } \\
& (\mathrm{mmol} / 1 \text { per } \mathrm{min}) \\
& \times \text { IAUC insulin }(\mathrm{mU} / 1 \text { per } \min ) / 22 \cdot 5 .
\end{aligned}
\end{aligned}
$$

This technique has been validated against the intravenous glucose tolerance test ${ }^{(10)}$.

\section{Statistical analyses}

From a previous study we had conducted, we postulated that the difference between the $12 \mathrm{~h}$ glucose area under the curve would be 90 with an SD of 70 assuming a power of 90 and an $\alpha$ of $0 \cdot 01$, and estimated the number of volunteers needed to be five ${ }^{(6)}$. Data were checked for normality and expressed as means with their standard errors. Differences were assessed by repeated-measures ANOVA and located with a Duncan's post hoc test.

\section{Results}

Interstitial glucose levels, obtained from the continuous glucose monitor for the four meal patterns are shown in Fig 1. The total area under the glucose response curve and the $2 \mathrm{~h}$ postprandial areas under the response curves for insulin, TAG and NEFA are shown in Table 3. Glucose and insulin responses showed broadly similar patterns. Both meal timing and quality of carbohydrate affected postprandial glucose and insulin responses $(P<0 \cdot 01)$. The area under the glucose and insulin response curves was greatest for the HGI-pm meal regimen. The HGI-pm meal regimen produced a significantly greater postprandial area under the glucose curve than for any of the other three meal regimens $(P<0.05)$. The postprandial area under the insulin curve was significantly greater than both the LGI regimens $(P<0 \cdot 05)$. Postprandial insulin resistance measured by homeostatic model assessment was also significantly greater for the HGI-pm meal than for the two LGI meals $(P<0 \cdot 05)$. Postprandial TAG and NEFA levels were not affected by meal timing or carbohydrate quality.

\section{Discussion}

It has been known for some time that low-GI foods lower the postprandial glycaemic response ${ }^{(11)}$ and low-GI foods taken throughout the day lower 12 and $24 \mathrm{~h}$ postprandial glycae$\operatorname{mia}^{(12)}$. To our knowledge, this is the first study that simultaneously explores (a) the effect of energy load and high- or low-GI diets given at different times of the day in a traditional 

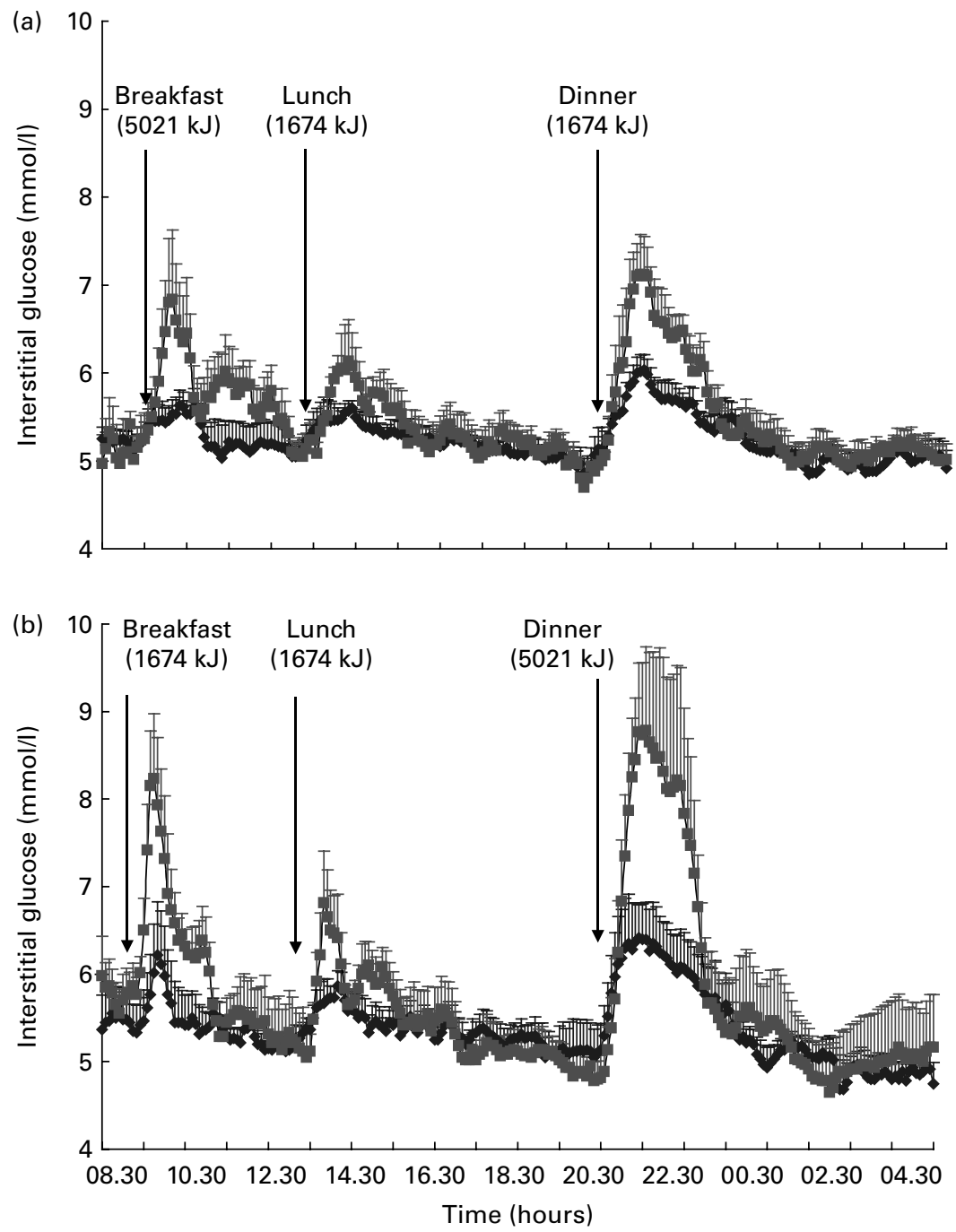

Fig. 1. Interstitial glucose levels in six healthy volunteers obtained every 5 min following either a high-glycaemic index (Gl) diet ( $\longrightarrow-$ ) or a low-Gl diet ( $\bullet-)$ with the majority of energy consumed either (a) early (early meal pattern) or (b) late (late meal pattern). Values are means with their standard errors represented by vertical bars. $5021 \mathrm{~kJ}$ is equivalent to $1200 \mathrm{kcal}$ and $1674 \mathrm{~kJ}$ is equivalent to $400 \mathrm{kcal}$.

three-meal eating pattern and (b) the effect of the quality of carbohydrate (in this case, GI) on postprandial metabolic responses. There were clear differences in circulating insulin and glucose when the large energy load was given in the evening compared to the morning, with the higher energy intake meal causing a significantly greater glucose and insulin response in the evening compared to the same meal consumed in the morning. This may be due to a relative insulin insensitivity in the evening, thought to be induced by increases in NEFA, although we were unable to demonstrate

Table 3. Total area under the curve for interstitial glucose $(0-20 \mathrm{~h})$, postprandial plasma insulin, TAG and NEFA (0-2h after each meal) in six healthy volunteers following either a high-glycaemic index (HGl) or a low-glycaemic index (LGI) diet, with most of the energy consumed either early (LGl-am, HGI-am) or late (LGI-pm, HGI-pm)

(Mean values with their standard errors)

\begin{tabular}{|c|c|c|c|c|c|c|c|c|c|c|}
\hline & \multicolumn{2}{|c|}{ Glucose $(\mathrm{mmol} / \mathrm{l} \times \mathrm{h})$} & \multicolumn{2}{|c|}{ Insulin $(\mathrm{pmol} / \mathrm{l} \times \mathrm{h})$} & \multicolumn{2}{|c|}{ HOMA-PP } & \multicolumn{2}{|c|}{ TAG $(\mathrm{mmol} / \mathrm{l} \times \mathrm{h})$} & \multicolumn{2}{|c|}{$\operatorname{NEFA}(\mathrm{mmol} / \mathrm{l} \times \mathrm{h})$} \\
\hline & Mean & SEM & Mean & SEM & Mean & SEM & Mean & SEM & Mean & SEM \\
\hline LGI-am & $104^{*}$ & 1.3 & $753^{*}$ & 134 & $6 \cdot 1^{*}$ & 1.4 & 6.03 & 0.85 & 2.54 & 0.28 \\
\hline HGI-am & $110^{*}$ & $1 \cdot 1$ & $951^{\dagger}$ & 188 & $17 \cdot 1$ & 3.9 & $6 \cdot 40$ & 0.46 & $2 \cdot 26$ & 0.29 \\
\hline LGI-pm & $106^{*}$ & 1.5 & $668^{*}$ & 156 & $6 \cdot 6^{\star}$ & $1 \cdot 8$ & $6 \cdot 20$ & 0.92 & $2 \cdot 34$ & 0.40 \\
\hline HGI-pm & 116 & 3.9 & 1142 & 230 & $24 \cdot 2$ & 5.6 & 5.04 & 0.79 & 2.49 & 0.51 \\
\hline
\end{tabular}

HOMA-PP, postprandial homeostatic model assessment.

${ }^{*}$ Mean value was significantly different from that of the HGI-pm condition $(P<0.05)$

† Mean value was significantly different from that of the LGI-pm condition $(P<0.05)$.

$\ddagger$ HOMA-PP is expressed in arbitrary units. 
significant differences in circulating NEFA levels between the meal regimens ${ }^{(13)}$. Another possible explanation is that the LGI-pm dietary pattern increases SCFA production from colonic fermentation and this reduces hepatic glucose production leading to improved insulin sensitivity, an observation that has been made by Thorburn et al. ${ }^{(14)}$. An insight into the effect of the four meal patterns on insulin sensitivity has been given by the HOMA-PP calculation. This suggests that the HGI-pm meal pattern has the most detrimental effect on insulin resistance over the day compared to both LGI meal patterns which would fit with the observations by Thorburn et al. ${ }^{(14)}$ discussed previously. In an earlier study where we measured day profiles of insulin and glucose during low- and high-GI diets, where the greatest energy load was given in the evening, we also demonstrated deterioration in insulin sensitivity during the high-GI diet ${ }^{(6)}$. The reasons for this are not understood at the present time.

The LGI meals lowered the impact of the high energy intake on glucose and insulin metabolism both in the morning and evening. This was most marked in the evening, lowering the AUC by approximately $50 \%$. The effect of lowering blood glucose is a fundamental aspect of low-GI diet. The mechanisms behind the metabolic effects are multifactorial and could include food matrix, decreased gastric emptying rate, slower small-intestinal absorption of glucose and an attenuated insulin response ${ }^{(7)}$. As reported previously, a low glycaemic intake lowered the total $24 \mathrm{~h}$ glucose regardless of whether the highenergy meal was taken in the morning or evening ${ }^{(6)}$.

We have previously shown an impaired postprandial lipid tolerance when meals are consumed at night, compared with the daytime ${ }^{(13)}$. TAG levels were unaffected by meal regimen in the present study; however, this is probably because the meals contained a relatively low percentage of fat, and blood sampling was only carried out for $2 \mathrm{~h}$ postprandially whereas peak postprandial TAG levels occur between 4 and $6 \mathrm{~h}$. It is of interest that the HGI-am diet has the same insulin response as the HGI-pm diet; however, the glucose response over the day is significantly lower with the HGI-am diet. This suggests that in the case of a HGI diet, the timing of the major energy load makes a significant impact on nutrient handling. This may be due to the metabolic differences occurring during the day, such as the increase in NEFA in the evening.

Our hypotheses around meal composition focused on the effect of the GI of the diets. Although we controlled the total energy, fat and protein, the dietary fibre was higher and the glycaemic load lower in the LGI group. This is impossible to avoid when using commonly consumed foods. It cannot be discounted that some of the effects are due to the increase in cereal-based fibres. A recent study has shown that inert cereal dietary fibre does have an effect on insulin sensitivity $^{(15)}$.

The present study reports the acute changes observed in the extremes of GI and meal patterns. The potential health benefits of an individual moving from their habitual high evening energy intake to a meal pattern where energy intake is greater in the morning with a low GI need to be assessed, as with the potential long-term benefits.
The traditional eating patterns in many Western societies of having the largest energy load in the evening may contribute to the metabolic syndrome through deterioration in postprandial glucose and insulin. This study indicates that avoidance of large, high-GI meals in the evening, in the context of a highGI diet, may be particularly beneficial in improving glucose profiles and may lead to reduced risk of type 2 diabetes and CHD.

\section{Acknowledgements}

The Nutrition and Dietetic Research Group is funded by grants from the MRC, BBSRC, NIHR, Diabetes UK, and funding from the NIHR Imperial Biomedical Research Centre Funding Scheme. G. F. is supported by an NIHR Senior Investigator award. All authors (L. M. M., J.-W. S., S. M. H. and G. F.) contributed to the development of the original idea, protocols and methods, analysis of results and the preparation of the manuscript. J.-W. S and S. M. H. carried out the study. The authors declare that there are no conflicts of interest.

\section{References}

1. Abdul-Ghani MA, Stern MP \& Lyssenko V (2010) Minimal contribution of fasting hyperglycemia to the incidence of type 2 diabetes in subjects with normal 2-h plasma glucose. Diabetes Care 33, 557-561.

2. Tenkanen L, Sjoblom T \& Harma M (1998) Joint effect of shift work and adverse life-style factors on the risk of coronary heart disease. Scand J Work Environ Health 24, 351-357.

3. Weickert MO, Roden M, Isken F, et al. (2011) Effects of supplemented isoenergetic diets differing in cereal fiber and protein content on insulin sensitivity in overweight humans. Am J Clin Nutr 94, 459-471.

4. van Cauter E (1990) Diurnal and ultradian rhythms in human endocrine function: a minireview. Horm Res 34, 45-53.

5. Lund J, Arendt J, Hampton SM, et al. (2001) Postprandial hormone and metabolic responses amongst shift workers in Antarctica. J Endocrinol 171, 557-564.

6. Brynes AE, Mark Edwards C, Ghatei MA, et al. (2003) A randomised four-intervention crossover study investigating the effect of carbohydrates on daytime profiles of insulin, glucose, non-esterified fatty acids and triacylglycerols in middle-aged men. Br J Nutr 89, 207-218.

7. Wolever TM (1990) The glycemic index. World Rev Nutr Diet 62, 120-185.

8. Salmeron J, Manson JE, Stampfer MJ, et al. (1997) Dietary fiber, glycemic load, and risk of non-insulin-dependent diabetes mellitus in women. JAMA 277, 472-477.

9. Raben A, Holst JJ, Madsen J, et al. (2001) Diurnal metabolic profiles after $14 \mathrm{~d}$ of an ad libitum high-starch, high-sucrose, or high-fat diet in normal weight never-obese and postobese women. Am J Clin Nutr 73, 177-189.

10. Caumo A, Bergman RN \& Cobelli C (2000) Insulin sensitivity from meal tolerance tests in normal subjects: a minimal model index. J Clin Endocrinol Metab 85, 4396-4402.

11. Jenkins DJ, Wolever TM, Collier GR, et al. (1987) Metabolic effects of a low-glycemic-index diet. Am J Clin Nutr $\mathbf{4 6}$, 968-975.

12. Brynes AE, Adamson J, Dornhorst A, et al. (2005) The beneficial effect of a diet with low glycaemic index on $24 \mathrm{~h}$ 
glucose profiles in healthy young people as assessed by continuous glucose monitoring. Br J Nutr 93, 179-182.

13. Morgan LM, Aspostolakou F, Wright J, et al. (1999) Diurnal variations in peripheral insulin resistance and plasma nonesterified fatty acid concentrations: a possible link? Ann Clin Biochem 36, 4, 447-450.
14. Thorburn A, Muir J \& Proietto J (1993) Carbohydrate fermentation decreases hepatic glucose output in healthy subjects. Metabolism 42, 780-785.

15. Weickert MO, Mohlig M, Schofl C, et al. (2006) Cereal fiber improves whole-body insulin sensitivity in overweight and obese women. Diabetes Care 29, 775-780. 